\title{
Mode Selection Strategy for Multi-Mode Power Converters to Minimize its Differential Power
}

\author{
R. Ramos, I. Zubitur, D. Serrano, J.A. Oliver, P. Alou, J.A. Cobos
}

\begin{abstract}
In this paper we propose the mode selection strategy of a multi-mode single-stage single-phase inverter and the associated mode selection scheme for double-line-frequency power pulsation decoupling between the input an the output port for PV (photovoltaic) panel applications.

Multiple modulation strategies are used along the line cycle to optimize the overall efficiency of the converter. The proposed topology uses several modulations for the inductor current waveform, such that the power handled internally by the elements in the converter is minimized at any time, while performing the output voltage control and power decoupling.

The evaluation and selection of the most appropriate modulation in real-time represents a challenge for the control. This work allows to reduce the computational cost of evaluating the decision metric.

A mode selection scheme is proposed and validated in a hardware prototype.
\end{abstract}

\section{INTRODUCTION}

In this paper we use the Differential Power metric [1], [2] to quantify the power processed by the reactive components. By applying at any time the mode which minimizes this metric, inductor and semiconductors usage is optimized and results in improved system efficiency and higher energy density.

It is possible to determine the best mode to be applied in each instant of the line-cycle computing the Differential Power for each possible modulation. The scheme used in this work overcomes the challenge of real-time differential power computation for every mode and optimum mode selection with a simple approach.

Using this approach, a flying capacitor multi-level (FCML) inverter is operated in multiple modes. By changing the shape of the inductor current, the same current is used to perform two functions simultaneously: energy transfer and energy storage.

The mode selection scheme for this topology is proposed, and the global behavior of the multi-mode converter is illustrated along the line-cycle with simulations and validated with experimental results.

\section{Multi-MODE CONVERTERS}

Some power topologies can be operated with several modulation modes achieving the same voltage and current at their ports with different power processing [3]-[7]. This has special interest in high power density converters.

\section{A. Example-Google Little Box Challenge (LBC)}

The general problem can be considered in terms of a threeport converter.

For the LBC single-phase inverter [8], the input port is a $400 V_{D C} \mathrm{PV}$ source, the output port is the $240 V_{r m s} \mathrm{AC}$ load. While the context of this example is PV DC source and AC load, other application cases with AC or DC ports are equivalent. Other examples are AC/DC converters with Power Factor correction.

Converters with a low input current ripple DC port and a single-phase AC port must store energy to buffer the inherent instantaneous power mismatch between the input an the output port with a double-frequency power storage port. The FCML inverter is operated in such a way that the two reactive components are involved in the power balance. A capacitor acting in line-cycle whose minimum volume is determined by the required energy storage and the discharge ratio and an inductor, as a high frequency storage element which will distribute the power among the input, output and storage capacitor. The inductor volume and losses depend on the average power processed by the inductor along the line cycle that is, the differential power processed by the inductor. The lower the $P_{\text {diff }}$ the lower the inductor volume.

The case of the switched converter under consideration, schematically presented in Fig.1, is based on the flying capacitor converter [9], with the distinctive feature that in this case, the flying capacitor is also used as the energy buffer component to handle the double-line-frequency power ripple. The bus voltage on the flying capacitor is regulated at $0.8 \mathrm{~V}_{g}$. In this topology, the charge balance in storage capacitor $\left(C_{s}\right)$ is not done at switching frequency but at line frequency. As is not needed to assure charge balance during a switching cycle, multiple modulations are used to control both output voltage, and input current. Using different modulation sequences along the line cycle makes the control more complex since the converter has a different plant for each modulation. A control scheme based on the plant inversion technique is used to control the converter. A control based on the plant inversion technique [10] has been used to be able to design very simple linear controllers that can be used in the modulations, which 


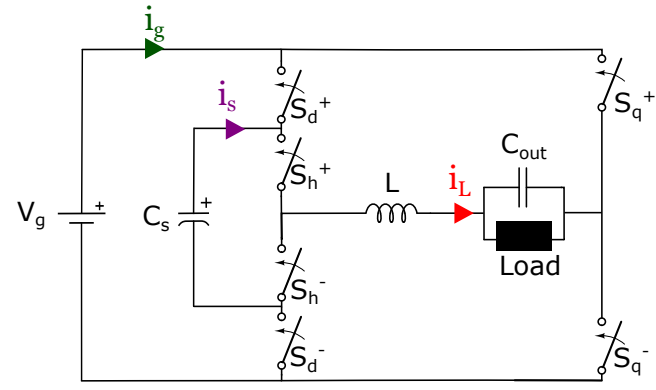

Figure 1. Simplified schematic of the flying capacitor inverter

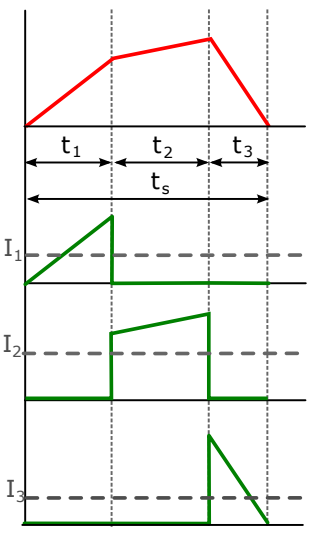

(a)

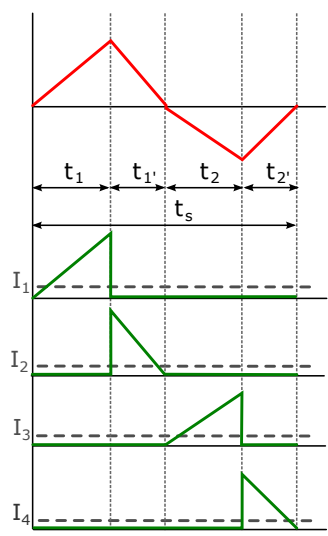

(b)
Figure 2. Trapezoidal and triangular modes

simplifies the control design.

At any instant along the line cycle, several modulations may obtain the same voltages and currents at the ports, but yielding very different losses due to different inductor and semiconductor usage.

Namely, there are up to 19 different switching modes, which can be classified depending on the inductor current waveform generated, in two types: trapezoidal modes Fig.2a and triangular modes Fig.2b.

While the inductor current will be the orange line, the input current and the storage capacitor current could be a combination of $I_{1}, I_{2}, I_{3}$ and for the triangular modes, also $I_{4}$. For this reason, with a trapezoidal mode the output current will always be higher than the input current, but with a triangular mode the input can be higher or lower than the output current.

In order to select the most appropriate mode at any instant, a decision criterion is needed. In this paper, Differential Power $\left(P_{d i f f}\right)$ is used as a metric to choose it [2]. The selected

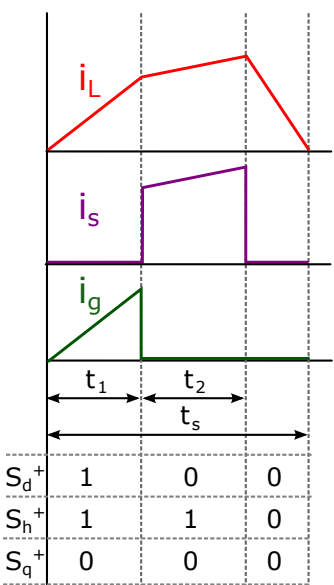

(a) mode IV

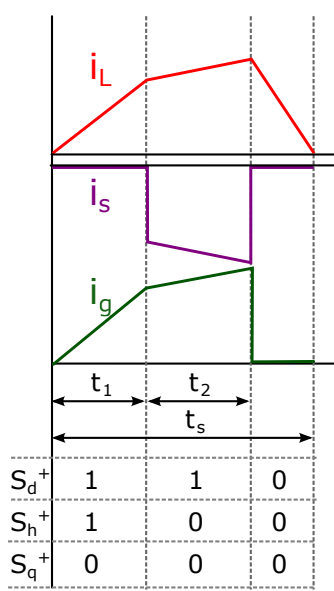

(b) mode VI
Figure 3. Trapezoidal modes

mode is the one with the lowest Differential Power among the modes that can be applied in each particular instant along the line-cycle. Since not all modes can operate throughout the whole line cycle, it is necessary to determine their regions of existence. As an example, comparing mode VI and mode IV, shown in Fig.3, considering positive storage capacitor current the one that discharges the capacitor, mode VI only charges the storage capacitor and mode IV only discharges it.

\section{DifFERENTIAL POWER ANALYSIS AND MODE SELECTION}

\section{A. Definition of the metric}

In a converter, the part of the output power which is directly transferred, and not processed by the reactive component, can be referred to as Direct Power $\left(P_{d i r}\right)$. The part of the output power which is processed by the reactive component can be referred to as "indirect power" or "internal power", being its minimum posible value the Differential Power $\left(P_{d i f f}\right)$ [2].

In the buck-boost family of converters, all the energy delivered to the load is previously stored in a magnetic component (inductor or flyback transformer). However, in most power converters (buck and boost families) there is a time interval in the switching period, in which the current is flowing directly from source to load. This current besides transferring energy directly from input to output, also stores energy in the inductor.

Applied to an inductor, Differential Power $\left(P_{d i f f}\right)$ is defined as the average power stored or delivered by the inductor.

$$
\begin{gathered}
P_{\text {diff } f_{L}}=\bar{i}_{L} \cdot V_{\text {charge } L} \cdot \frac{t_{\text {charge }}}{T} \\
V_{\text {chargeL }}=V_{g}-V_{o} ; \bar{i}_{L}=I_{o} ; t_{\text {charge }}=T_{\text {on }} ; T=T_{s} ;
\end{gathered}
$$

$P_{d i f f}$ in a buck converter inductor is:

$$
P_{\text {diff }}=I_{o}\left(V_{g}-V_{o}\right) d=P_{o}(1-d)
$$




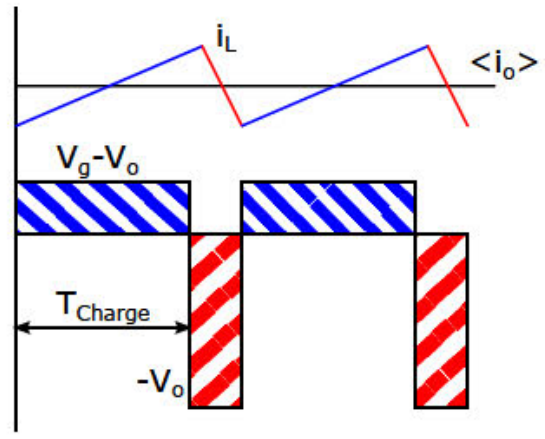

Figure 4. Buck converter inductor waveforms and $P_{\text {diff }}$

\section{B. Time domain analysis of the multi-mode inverter}

Along the line-cycle, certain values of $\left\{v_{s}, i_{s}, v_{o}, i_{o}, i_{g}, v_{g}\right\}$ must be obtained at the ports as illustrated in Fig.5b.

The converter operates in BCM (boundary conduction mode), and because of that, the averaged current through the inductor and the input current for every specific pattern $\mathrm{k}$, will depend on the two independent times, $t_{1}$ and $t_{2}$, the state $x=\left(v_{s}, v_{o}\right)$ and the input voltage, algebraically according to:

$$
\begin{aligned}
& <i_{o}>=f_{k}\left(t_{1}, t_{2}, x, V_{g}\right) \\
& <i_{g}>=g_{k}\left(t_{1}, t_{2}, x, V_{g}\right)
\end{aligned}
$$

In principle, for a given reference input current and reference output current, it will be possible to determine if for the sequence $k$ there exists a solution, and, in that case, to determine $t_{1}$ and $t_{2}$ that will provide the desired values for the input and output current.

For every instant of the line-cycle ( $\mathrm{x}$ axis), there are several modes available (each colour) which achieve exactly the same objective, but with great variation of the internal power $P_{\text {diff }}$ (sometimes a factor of 3 as in $\frac{\omega t}{\pi}=0.1$ ).

Selecting the mode with the lowest $P_{\text {diff }}$ in every instant, the plot gives the sequence in which the converter operates processing the minimum envelope of $P_{\text {diff }}$, thus minimizing the fraction of output power processed internally.

For this converter modulation, the average $P_{\text {diff }}$ results in 0.2 of $P_{o}$ processed. Comparatively, an inverter with an active filter and a discharge ratio of $40 \%$ in the storage capacitor would result in $P_{\text {diff }}=0.4$, which is twice the value of $P_{\text {diff }}$ obtained in this single stage inverter.

In bold line in Fig.5a, the minimum differential power envelope obtained by the optimum mode sequence is shown. Each a marker is a change in the operation mode.

This time domain analysis yields the minimum $P_{\text {diff }}$ power handling mode sequence inside a line-cycle, for a particular resistive load. However, $P_{\text {diff }}$ of each mode and thus the optimum mode sequence varies in real-time, with load-steps,

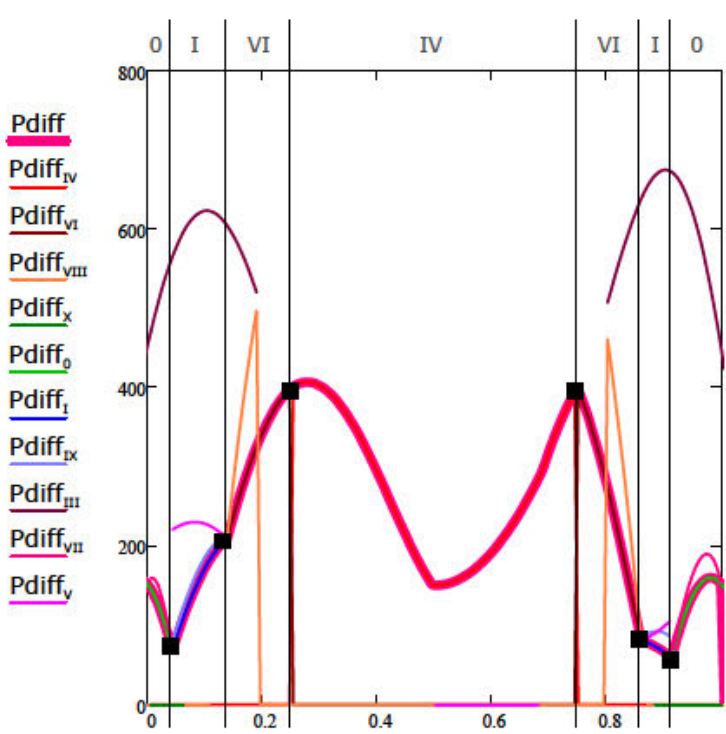

(a)

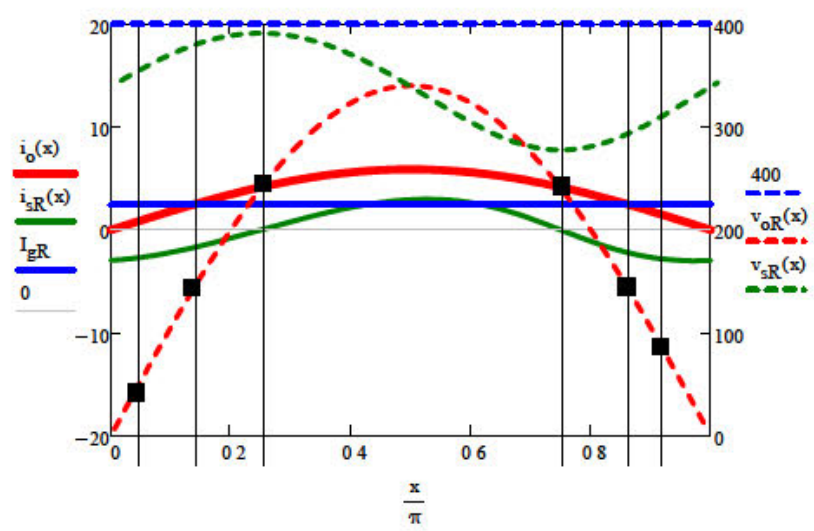

(b)

Figure 5. (a): $P_{\text {diff }}$ plot for every modulation along the line-cycle. Each color corresponds to one mode. In bold line, minimum $P_{\text {diff }}$ envelope achieved by the optimum mode sequence. (b)Ports currents and voltages along the linecycle. Currents in solid line, with its corresponding element voltage dashed.

power factor of the load, or due to control restrictions during transients. This depicts the need to develop a Mode Selection strategy valid for real-time implementation in a converter.

\section{IMPLEMENTATION (STATE-PLANE TRAJECTORY ANALYSIS)}

Real-time computing of $P_{\text {diff }}$ for all possible operating modes, and selection of the optimum in a microcontroller, is not feasible to implement. It is therefore necessary to implement a real time mode-selection scheme.

First, the operating restrictions for each mode are derived. Most of the modulation boundaries correspond to restrictions of power flow between ports (i.e. $i_{o}>i_{g}, i_{s}>i_{g}$ ) and voltage levels. 
For the mode IV shown in Fig.3a to be applicable, $t_{1}$ and $t_{2}$ must be grater than zero and voltage applied to the inductor (slopes) $m_{1}, m_{2}$ and $m_{3}$ needs to satisfy:

$$
m_{1}>0 ; m_{3}>0 ; m_{2} \text { either }
$$

The averaged current through the inductor and the input current for this particular mode can be calculated as:

$$
\begin{aligned}
<i_{o}>= & \frac{\frac{1}{2} m_{1}\left(1+\frac{m_{1}}{m_{3}}\right) t_{1}^{2}+\frac{1}{2} m_{2}\left(1+\frac{m_{2}}{m_{3}}\right) t_{2}^{2}}{t_{1}\left(1+\frac{m_{1}}{m_{3}}\right)+t_{2}\left(1+\frac{m_{2}}{m_{3}}\right)}+ \\
& +\frac{m_{1}\left(1+\frac{m_{2}}{m_{3}}\right) t_{1} t_{2}}{t_{1}\left(1+\frac{m_{1}}{m_{3}}\right)+t_{2}\left(1+\frac{m_{2}}{m_{3}}\right)} \\
& <i_{g}>=\frac{\frac{1}{2} m_{1}\left(1+\frac{m_{1}}{m_{3}}\right) t_{1}^{2}}{t_{1}\left(1+\frac{m_{1}}{m_{3}}\right)+t_{2}\left(1+\frac{m_{2}}{m_{3}}\right)}
\end{aligned}
$$

To reduce the number of variables to represent both the current and the output voltage are normalized with respect to the values of the input port. In this way the normalized output current is:

$$
i_{\text {on }}=\frac{\left\langle i_{o}\right\rangle}{\left\langle i_{g}\right\rangle}
$$

Substituting (7) and (8) in (9)

$$
\begin{aligned}
i_{\text {on }}= & \frac{\frac{1}{2} m_{1}\left(1+\frac{m_{1}}{m_{3}}\right) t_{1}^{2}+\frac{1}{2} m_{2}\left(1+\frac{m_{2}}{m_{3}}\right) t_{2}^{2}}{\frac{1}{2} m_{1}\left(1+\frac{m_{1}}{m_{3}}\right) t_{1}^{2}}+ \\
+ & \frac{m_{1}\left(1+\frac{m_{2}}{m_{3}}\right) t_{1} t_{2}}{\frac{1}{2} m_{1}\left(1+\frac{m_{1}}{m_{3}}\right) t_{1}^{2}}
\end{aligned}
$$

And solving (10)

$$
t_{1}=\left\{\begin{array}{l}
-\frac{t_{2}\left[\sqrt{\frac{m_{3}}{m_{1}}\left(m_{2}+m_{3}\right)\left(m_{1}+m_{2}\left(i_{o n}-1\right)\right)}+m_{2}+m_{3}\right]}{m_{1}+m_{3}\left(1-i_{o n}\right)} \\
-\frac{t_{2}\left[-\sqrt{\frac{m_{3}}{m_{1}}\left(m_{2}+m_{3}\right)\left(m_{1}+m_{2}\left(i_{o n}-1\right)\right)}+m_{2}+m_{3}\right]}{m_{1}+m_{3}\left(1-i_{o n}\right)}
\end{array}\right.
$$

Knowing that for this particular mode

$$
\begin{gathered}
m_{1}=\frac{V_{g}-v_{o}}{L} \\
m_{2}=\frac{v_{s}-v_{o}}{L} \\
m_{3}=\frac{v_{o}}{L}
\end{gathered}
$$

the mode IV boundaries are:

$$
\begin{aligned}
& v_{o n}<1 \\
& i_{o n}>1 \\
& i_{o n}>\frac{1}{v_{o n}} \\
& i_{\text {on }}<\frac{v_{s n}-1}{v_{s n}-v_{o n}} v_{s n}-v_{o n}>0
\end{aligned}
$$

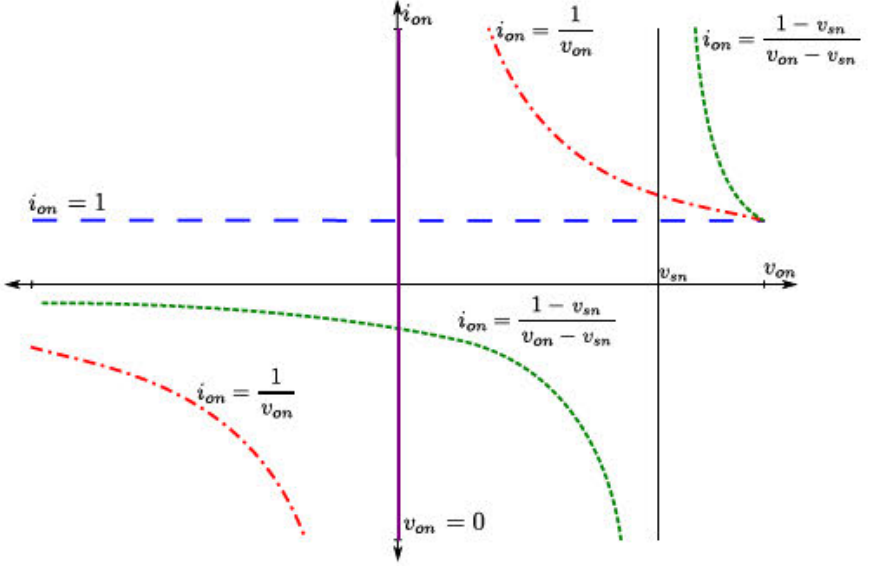

Figure 6. Mode IV boundaries

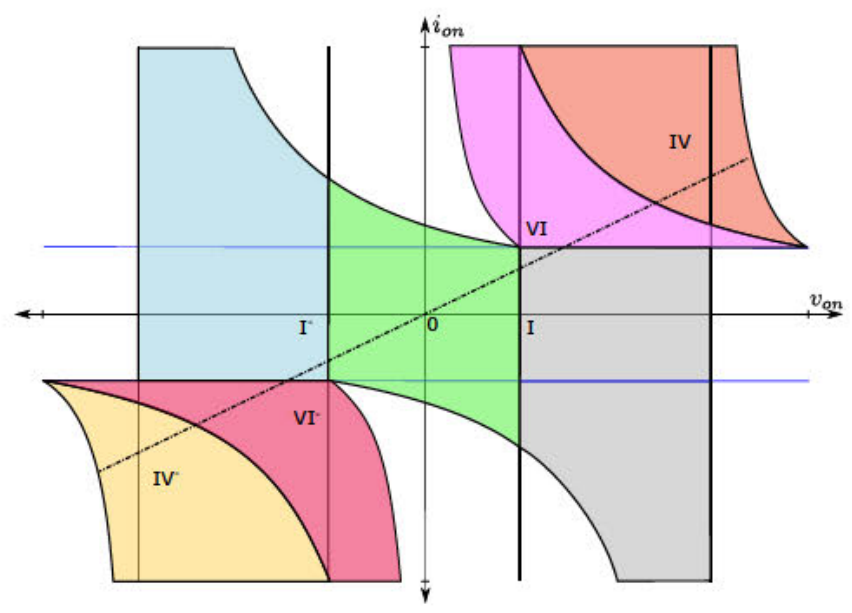

Figure 7. Mode boundaries superimposed to the operating point trajectory for resistive load.

$$
i_{\text {on }}>\frac{v_{s n}-1}{v_{s n}-v_{\text {on }}} v_{s n}-v_{\text {on }}<0
$$

These boundaries, represented in Fig.6, are in the form of simple inequations, which are very easily implemented in a microcontroller and suitable for real time evaluation of the modes. Performing the same analysis for all the modes, the conditions of existence of all modes can be obtained, in Table I the boundary conditions for resistive modes are summarized. And plotting the resulting restrictions, the state space is divided in several areas. In areas where more than one mode can be applied, the one with the lowest $P_{\text {diff }}$ is chosen.

This state-space is represented in two normalized axis: $\frac{i_{o}}{I_{g}}$, the vertical axis where $i_{o}$ is the output current; $\frac{v_{o}}{V_{g}}$, where $v_{o}$ is the output voltage of the inverter. The mode change points identified in the time domain analysis of Fig.5, now correspond to mode boundary crossings in Fig.7.

Based on the state-space shown in Fig.7, a decision tree (Fig.8) is implemented on the microcontroller to select the 
Table I

EXISTENCE CONDITIONS FOR RESISTIVE MODES

\begin{tabular}{|c|c|}
\hline Mode & Conditions \\
\hline 0 & $\begin{array}{c}v_{s n}-1<v_{o n}<1-v_{s n} \\
-V_{s n}<V_{o n}<V_{s n} \\
\frac{1}{v_{o n}-v_{s n}}<i_{o n}<\frac{1}{v_{o n}+v_{s n}}\end{array}$ \\
\hline I & $\begin{array}{c}1-V_{s n}<V_{o n}<V_{s n}-1 \\
v_{o n}<1 \\
v_{o n}<V_{s n} \\
i_{o n}<1 \\
i_{o n}>\frac{1}{v_{o n}-v_{s n}}\end{array}$ \\
\hline VI & $\begin{array}{c}v_{o n}>0 \\
v_{o n}<1 \\
i_{o n}>1 \\
i_{o n}<\frac{1}{v_{o n}} \\
i_{o n}>\frac{1-v_{\text {on }}}{v_{o n}} \\
\end{array}$ \\
\hline IV & $\begin{aligned} & v_{o n}>0 \\
& v_{o n}<1 \\
& i_{o n}>1 \\
& i_{o n}>\frac{1}{v_{o n}} \\
& i_{o n}<\frac{v_{s n}-1}{v_{s n}-v_{o n}} v_{s n}-v_{o n}>0 \\
& i_{o n}>\frac{v_{s n}-1}{v_{s n}-v_{o n}} v_{s n}-v_{o n}<0\end{aligned}$ \\
\hline
\end{tabular}

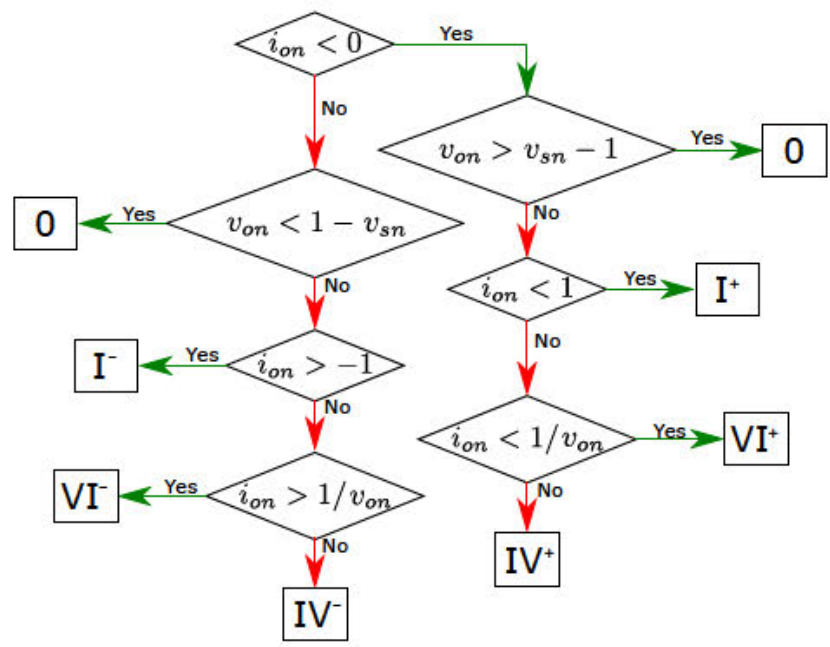

Figure 8. Mode selector diagram

optimum mode. Using the same procedure described above for resistive loads the boundaries for the zones of inductive/capacitive loads have been obtained. The new state-space valid for all the load types is shown in Fig.9

\section{IMPACT OF NON-IDEALITIES}

The mode boundaries shown above have been plotted for a constant storage capacitor voltage. However, to reduce volume,

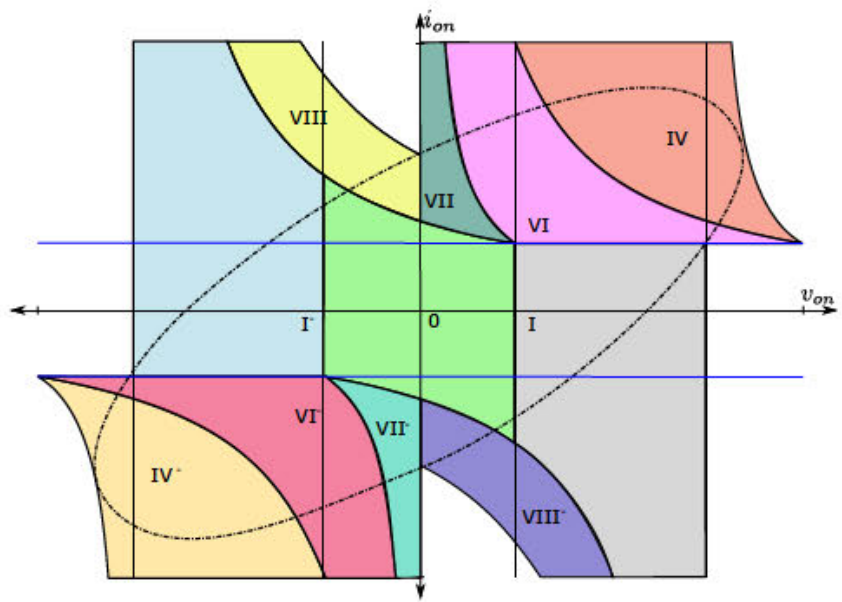

Figure 9. Mode boundaries superimposed to the operating point trajectory for capacitive and inductive load.

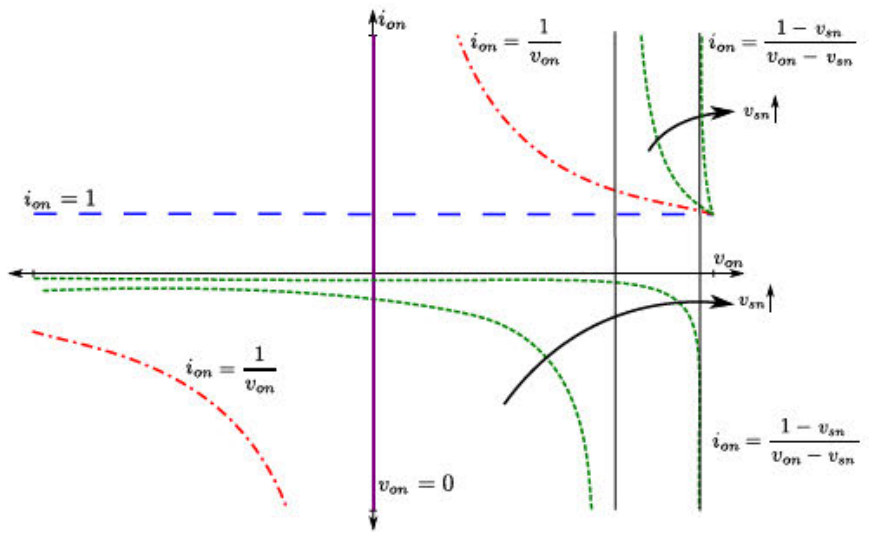

Figure 10. Boundaries' dependence of storage capacitor voltage.

the storage capacitor has a large discharge ratio. For this reason, the boundaries are not fixed but depend on the value of the voltage of the storage capacitor, as shown in Fig.10.

It should be noted that to achieve Zero Voltage Switching (ZVS), it is necessary to have a minimum current to discharge the parasitic capacitance of the switching devices, therefore the switching cycle starts not at zero inductor current but a $+I_{t h}$ or $-I_{t h}$ depending on the mode applied. In our case, the minimum current to achieve ZVS has been set at $\pm 2 A$.

Using the mode with the minimum differential power it is not always feasible. To limit the minimum switching frequency additional modes should be used. As shown in Fig.11 at the boundary between modes 0 and $I$ the switching frequency reach $0 \mathrm{~Hz}$ at $0.045 \pi$ and $0.91 \pi$. Around that boundary an additional mode (mode $I I I$ ) is used with a higher switching frequency but also with a higher differential power.

Furthermore, after a load step, the storage capacitor voltage diverts slightly from its operating point.

All of that leads the converter not to follow exactly the 


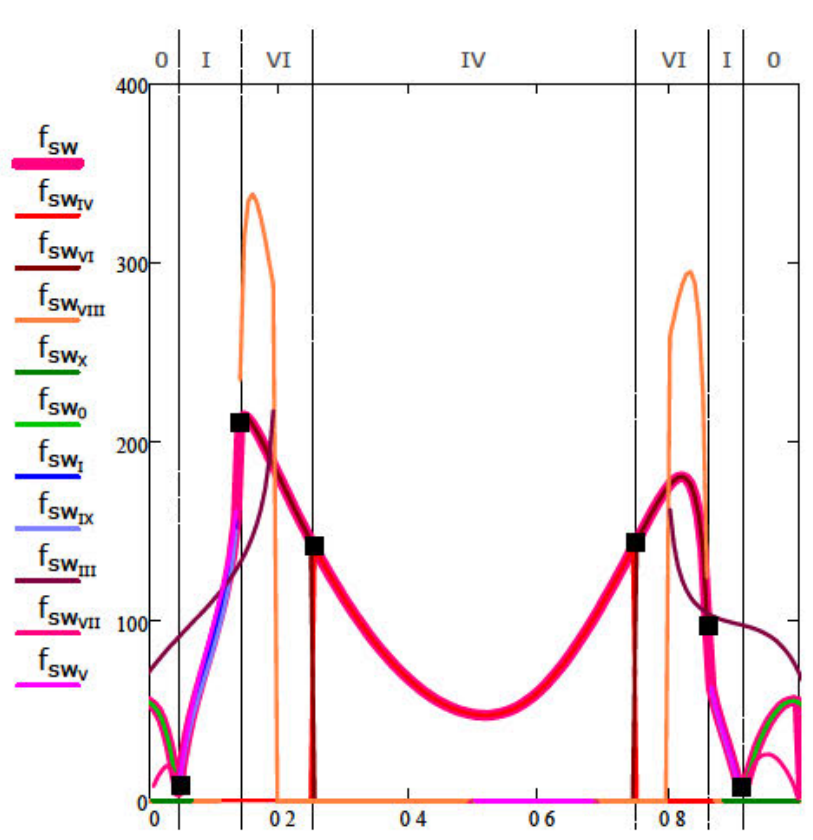

Figure 11. $f_{s w}$ plot for every modulation along the line-cycle. Each color corresponds to one mode. In bold line, $f_{s w}$ envelope achieved by the optimum mode sequence.

theoretical trajectory. As shown in Fig.12, the effect of nonidealities is more pronounced near the zero crossing.

\section{SIMULATION AND EXPERIMENTAL RESULTS}

In order to validate the proposed mode selection strategy, many simulations have been carried out. Not only with electrical simulator such us Gecko Circuit [11] with which Fig.13 was obtained, but also the system behavior was simulated within the FPGA (Fig.14) with the HDL simulator ModelSim [12], to prove that the algorithm works correctly on the actual system in which it is implemented.

Experimental results shown in Fig.15 validate the concept. The modulation selection scheme is implemented and validated in a low voltage hardware prototype, generating a $24 V_{r m s}, 60 \mathrm{~Hz}$, waveform, from a $40 V_{D C}$ input voltage, that is 10 times lower voltage than the initial specs, and nominal current. A new inverter for nominal voltage is being developed using high dv/dt drivers for high speed $\mathrm{GaN}$ devices to scale $V_{g}$ to $400 V_{D C}$. The waveforms shown in Fig. 15 are the mode number in yellow and the output voltage in blue.

The mode applied shown in Fig.15 closely matched that of the simulation at the bottom of Fig.13. With the difference that in this case the experimental waveform of the applied mode is very symmetric because it is using a higher capacitance in storage capacitor than the specified allowing a much smaller voltage ripple. A larger storage capacitor is used in order to represent more clearly the sequence of modes, although the

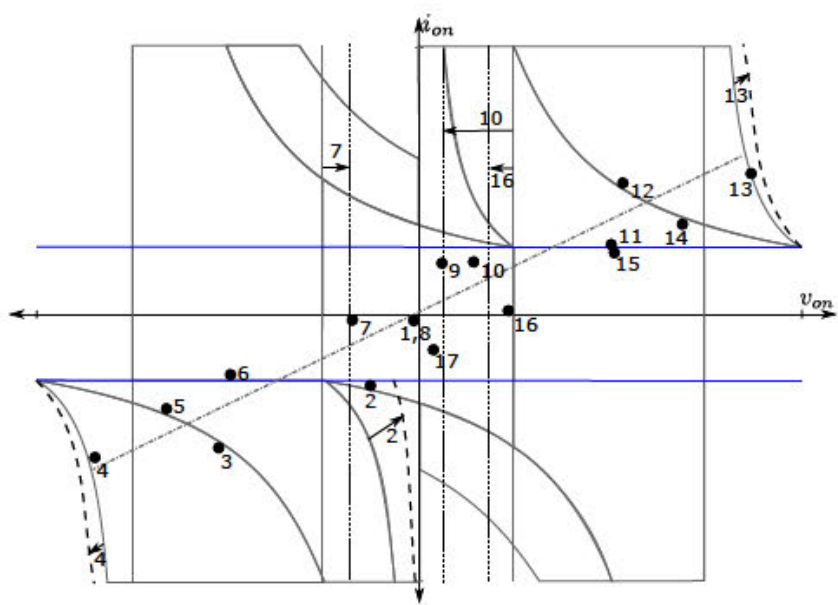

(a)

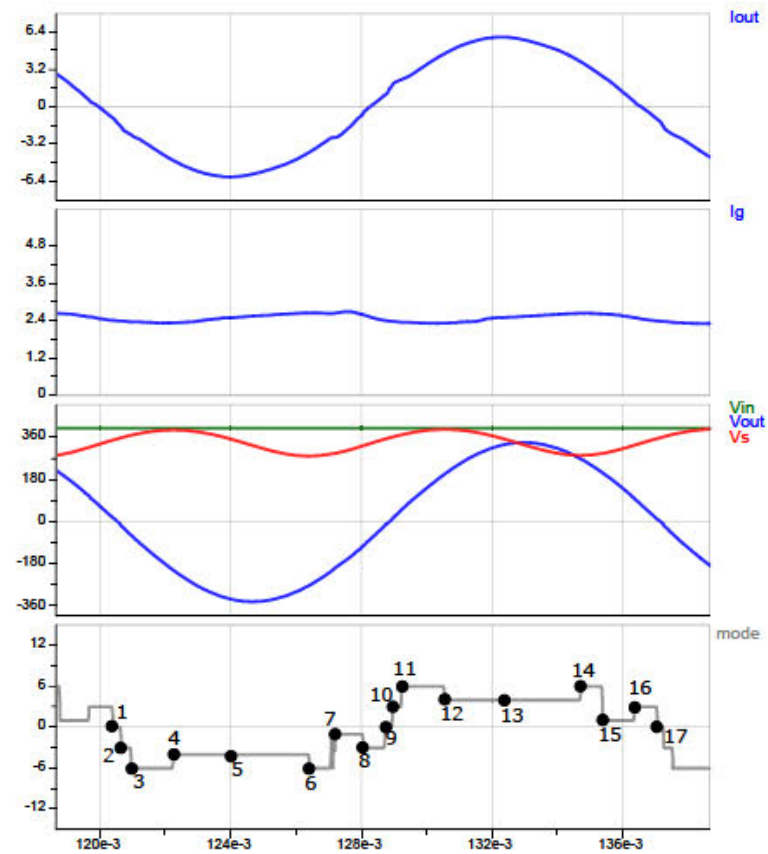

(b)

Figure 12. Time domain simulation illustrating the changes in the operation mode

whole system operates with a $40 \%$ discharge ratio of storage capacitor.

\section{CONCLUSION}

It is shown that a given power topology can be operated in multiple modes, for the same voltage and current in its three ports. Multiple modulation strategies are used along the line cycle to optimize the overall size and efficiency of the converter. Using the Differential Power as a metric, at any given time along the line cycle, the mode-selection scheme 


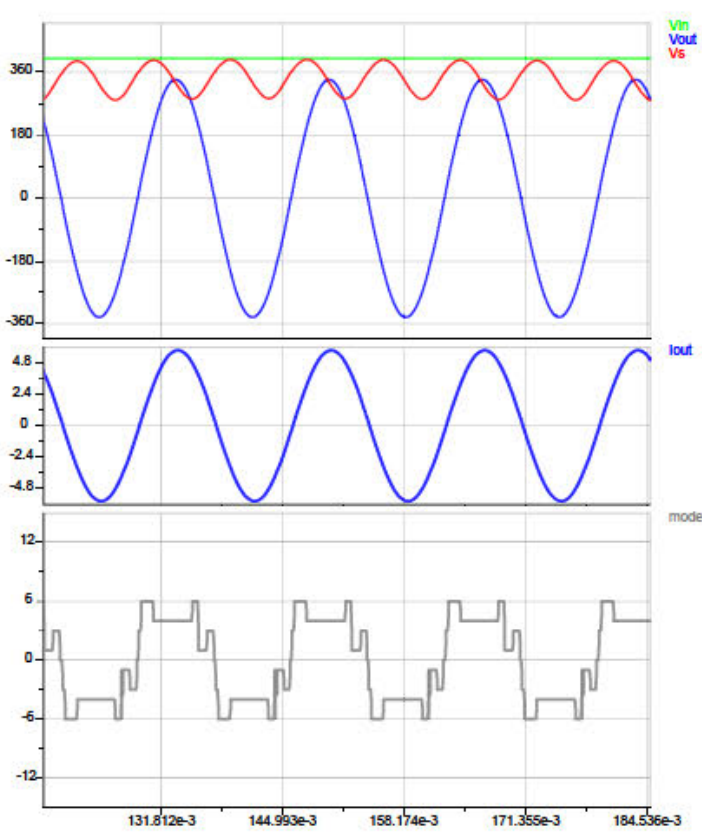

Figure 13. Simulated waveforms of the inverter: (Upper): Output voltage (blue) input voltage (green) and storage capacitor (red),(Middle) Output current, (Lower) Sequence number applied

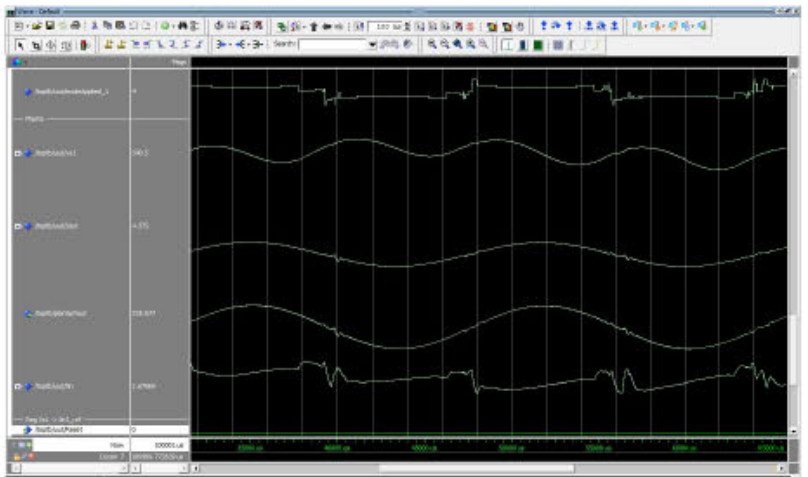

Figure 14. Simulated waveforms of the VHDL code. From top to bottom: mode applied, storage capacitor voltage, output current, output voltage and input current

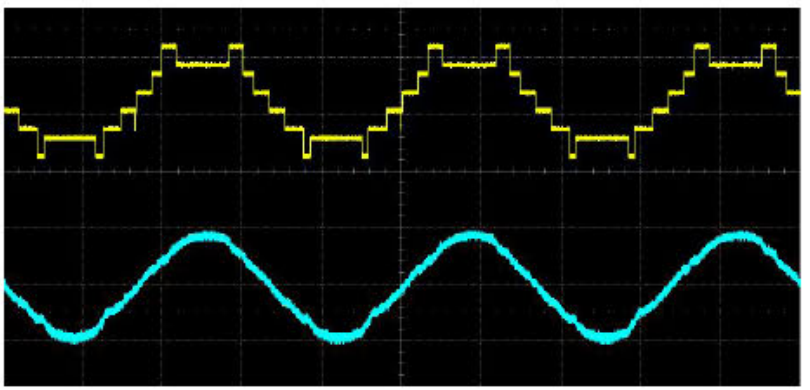

Figure 15. Experimental waveforms of the inverter: Output voltage (blue) Sequence number (yellow) presented applies the available mode which minimizes it in real time.

Based on the time-domain analysis and a derived analytical model of each modulation, the state-space for the converter is obtained and validated in a prototype converter.

\section{ACKNOWLEDGMENT}

The authors would like to thank the Spanish Ministry of Education, Culture and Sport for its support under the FPU grant program (Reference FPU15/07081).

\section{REFERENCES}

[1] J. A. Cobos, "Optimization of energy buffered converters," in Power Electronics (CIEP), 2016 13th International Conference on, IEEE, 2016.

[2] J. A. Cobos, H. Cristobal, D. Serrano, R. Ramos, J. A. Oliver, and P. Alou, "Differential power as a metric to optimize power converters and architectures," pp. 1-7.

[3] G. C. Christidis, A. C. Nanakos, and E. C. Tatakis, "Hybrid discontinuous/boundary conduction mode of flyback microinverter for ac-pv modules," IEEE Transactions on Power Electronics, vol. 31, no. 6, pp. 4195-4205, 2016.

[4] E. Alidori, G. Cimini, G. Ippoliti, G. Orlando, and M. Pirro, "A passivity-based solution for $\mathrm{ccm}$-dcm boost converter power factor control," in Industrial Electronics Society, IECON 2013-39th Annual Conference of the IEEE, pp. 7752-7757, IEEE, 2013.

[5] A. Fernández, H. Carbonnier, O. Mourra, and F. Tonicello, "Large signal model with dynamic ccm-dcm detection," in Energy Conversion Congress and Exposition (ECCE), 2012 IEEE, pp. 1585-1591, IEEE, 2012.

[6] S. F. Lim and A. M. Khambadkone, "A simple digital dcm control scheme for boost pfc operating in both $\mathrm{ccm}$ and dcm," IEEE Transactions on Industry Applications, vol. 47, no. 4, pp. 1802-1812, 2011.

[7] D.-S. Chen and J.-S. Lai, "A study of power correction boost converter operating at ccm-dcm mode," in Southeastcon'93, Proceedings., IEEE, pp. 6-p, IEEE, 1993.

[8] L. B. Challenge, "Detailed inverter specifications, testing procedure, and technical approach and testing application requirements for the little box challenge," https://www.littleboxchallenge.com/pdf/LBCInverterRequirements-20141216.pdf [Online: accessed 18-JAN-2015], 2015.

[9] T. Meynard and H. Foch, "Multi-level conversion: high voltage choppers and voltage-source inverters," in Power Electronics Specialists Conference, 1992. PESC'92 Record., 23rd Annual IEEE, pp. 397-403, IEEE, 1992.

[10] R. Ramos, D. Serrano, A. J. Oliver, and J. A. Cobos, "Control of a single phase inverter with multiple modulation strategies based on plant inversion," pp. 1-7, 2017.

[11] A. Musing and J. W. Kolar, "Successful online education-geckocircuits as open-source simulation platform," in Power Electronics Conference (IPEC-Hiroshima 2014-ECCE-ASIA), 2014 International, pp. 821-828, IEEE, 2014.

[12] M. Graphics, "Modelsim pe student edition," 2015. 